\title{
CDISC SDTM Common Terminology Criteria for Adverse Event Grade Terminology V3.0
}

National Cancer Institute

\section{Source}

National Cancer Institute. CDISC SDTM Common Terminology Criteria for Adverse Event

Grade Terminology V3.0. NCI Thesaurus. Code C66784.

Terminology codelist used with Common Criteria for Adverse Event Grade within the

Clinical Data Interchange Standards Consortium Study Data Tabulation Model. 